\title{
Diversity oriented approach to polycyclics via cross-enyne metathesis and Diels-Alder reaction as key steps
}

\author{
SAMBASIVARAO KOTHA*, VITTAL SEEMA, SHAIBAL BANERJEE and \\ MRITYUNJAY KUMAR DIPAK \\ Department of Chemistry, Indian Institute of Technology Bombay, Powai, Mumbai, Maharashtra, \\ 400 076, India \\ e-mail: srk@chem.iitb.ac.in
}

MS received 28 April 2014; revised 9 August 2014; accepted 11 August 2014

\begin{abstract}
Polycyclics containing biaryls have been prepared via application of cross-enyne metathesis and the Diels-Alder reaction. Here, ethylene and 1,5-hexadiene were used as a cross-metathesis partners to generate key diene intermediates.
\end{abstract}

Keywords. Diversity-oriented synthesis; aryl acetylenes; metathesis; Diels-Alder reaction; $\alpha$-amino acids.

\section{Introduction}

Polycyclics are useful entities in several areas of chemical sciences. ${ }^{1}$ Since a major portion of the present work described here deals with biaryl derivatives, we include a brief introduction to this interesting class of molecules. ${ }^{2}$ Biaryl-containing compounds (figure 1) are useful in material science, pharmaceuticals, polymers ${ }^{3}$ and ligands for catalysis. Classical methods for the synthesis of biaryls are based on cross-coupling (e.g., Suzuki, ${ }^{4}$ Stille,${ }^{5}$ Negishi ${ }^{6}$ and Kharasch coupling ${ }^{7}$ of two aromatic moieties in the presence of a transitionmetal catalyst. However, in some instances, these methods suffer from at least one of the following drawbacks: low yields, scrambling products and hydrolysis product, etc. Therefore, development of an alternative strategy to highly functionalized biaryls is warranted. For example, non-transition metal based methods ${ }^{8}$ such as DielsAlder (DA) cycloaddition is an alternative for the construction of a variety of biaryls because a wide range of structurally diverse biaryls can be assembled via this approach by varying the diene and dienophile components.

\section{Experimental}

\subsection{Materials and characterization}

Melting points were recorded on Lab hosp or Veego melting point apparatus. Infrared (IR) spectra were recorded on Nicolet Impact-400 FTIR spectrometer in

\footnotetext{
*For correspondence
}

$\mathrm{KBr} / \mathrm{CHCl}_{3} .{ }^{1} \mathrm{H} \mathrm{NMR}\left(300\right.$ and $400 \mathrm{MHz}$ ), ${ }^{13} \mathrm{C} \mathrm{NMR}$ (75 and $100.6 \mathrm{MHz}$ ) spectral data were determined at RT on a Varian VXR 400 in $\mathrm{CDCl}_{3}$ solution. Coupling constants ( $J$ values) are given in Hertz $(\mathrm{Hz})$. The highresolution mass measurements were carried out using Micromass Q-ToF spectrometer. Analytical thin layer chromatography (TLC) was performed on $(10 \times 5 \mathrm{~cm})$ glass plates coated with Acme's silica gel G or GF 254 (containing $13 \%$ calcium sulphate as a binder). Silica gel was coated on glass plates using 'Sandwich Technique'. Column chromatography was performed using Acme's silica gel (100-200 mesh) using double spray bellows for application of pressure and the column was eluted with ethyl acetate-petroleum ether mixture.

2.1a General procedure for the preparation homo allyl dienes: To a degassed solution of 4-ethynyl-1,2dimethoxybenzene $10(100 \mathrm{mg}, 0.61 \mathrm{mmol})$ and 1,5hexadiene (100 mg, $1.22 \mathrm{mmol})$ in toluene $(10 \mathrm{~mL})$ was added Grubbs second generation catalyst $(20 \mathrm{mg}$, $4 \mathrm{~mol} \%$ ) and the reaction mixture heated at $90^{\circ} \mathrm{C}$ for $12 \mathrm{~h}$. At the conclusion of the reaction (TLC monitoring), the reaction mixture was concentrated and the crude product was purified by a flash column chromatography using petroleum ether (PE) as an eluent to afford $\mathbf{1 1}$ (110 mg, 73\%) as a colourless liquid.

2.1b General procedure for the Diels-Alder reaction of homo allyl butadiene derivatives with napthoquinone: To a solution of $24(50 \mathrm{mg}, 0.162 \mathrm{mmol})$ in toluene (10 mL), 1,4-napthoquinone (25.5 mg, $0.162 \mathrm{mmol}$ ) was added and heated at $100^{\circ} \mathrm{C}$. At the conclusion of 
<smiles>[R]C(c1cc(-c2ccc(O)c(C[C@H](N)C(=O)N[C@@H](NC(=O)[C@H](C[C@@H](O)CN)C(=O)O)C(=O)O)c2)ccc1O)[C@@H](N)CN</smiles>

Biphenomycin $\mathrm{A} \mathrm{R}=\mathrm{OH}$ Biphenomycin B R=H<smiles>Pc1ccccc1OCCCOc1cccc(P)c1-c1ccccc1P</smiles>

2 Tunephos<smiles>O=C(O)Cc1ccc(-c2ccccc2)cc1</smiles>

3 Felbinac<smiles>COc1cc2c(c(OC)c1OC)-c1cc3c(cc1[C@@H](OC(C)=O)C1COC(=O)[C@H]1C2)OCO3</smiles>

Steganacin<smiles>Cc1cc(O)c2c(c1)C(=O)c1cc(O)c(-c3c(O)ccc4oc5cccc(O)c5c(=O)c34)c(O)c1C2=O</smiles>

Euxanmodin B<smiles>CC(=O)c1c(O)cc(O)c(-c2c(C)cc(O)c3c2C(=O)c2cc(O)cc(O)c2C3=O)c1O</smiles>

Kniphoione

Figure 1. Various biaryl containing molecules.

the reaction ( $14 \mathrm{~h}$, TLC monitoring), solvent was evaporated and the residue was charged on a silica gel column. Elution of the column with 5\% ethyl acetate/petroleum ether gave the desired product $\mathbf{3 1}$ as a yellowish thick liquid (40 mg, 52\%).

\section{1c General procedure for the Diels-Alder reaction} of homo allyl butadiene derivatives followed by aromatization: To a solution of the diene 21 (115 mg, $0.43 \mathrm{mmol})$ in toluene $(5 \mathrm{~mL})$ was added dimethyl acetylenedicarboxylate $(124 \mathrm{mg}, 0.87 \mathrm{mmol})$ and the reaction mixture was heated at $90^{\circ} \mathrm{C}$ for $24 \mathrm{~h}$. At the conclusion of the reaction (TLC monitoring), the solvent was concentrated at reduced pressure and the crude product obtained was purified by a silica-gel column chromatography, using EtOAc/PE to afford the DA adduct as a semi solid. Later, aromatization of the DA adduct was carried out with DDQ (153 mg, $0.67 \mathrm{mmol})$ in refluxing toluene $(10 \mathrm{~mL})$ for $30 \mathrm{~h}$. Then, the solvent was removed at reduced pressure and the crude product was purified by flash silica gel column chromatography by using (EtOAc/PE) mixture to afford the aromatized product $\mathbf{2 8}(128 \mathrm{mg}, 72 \%)$ as a semi-solid.

2.1d General procedure for the preparation of 1,3dienes and Diels-Alder reaction followed by aromatization: A solution of $\mathbf{1 0}(100 \mathrm{mg}, 0.61 \mathrm{mmol})$ in toluene $(10 \mathrm{~mL})$ was degassed with $\mathrm{N}_{2}$ for $10 \mathrm{~min}$ and then the Grubbs second generation catalyst $(26 \mathrm{mg}$, $5 \mathrm{~mol} \%$ ) was added. The reaction mixture was stirred under ethylene atmosphere ( $1 \mathrm{~atm}$, balloon pressure) and stirred at RT for $24 \mathrm{~h}$. At the conclusion of the reaction (TLC monitoring), the solvent was concentrated and the crude product was purified by a flash silicagel column chromatography using EtOAc/PE to afford $32(85 \mathrm{mg}, 73 \%)$ as a brownish liquid. Later, the diene $32(95 \mathrm{mg}, 0.5 \mathrm{mmol})$ in toluene $(10 \mathrm{~mL})$ was added dimethyl acetylenedicarboxylate $(142 \mathrm{mg}, 1.0 \mathrm{mmol})$ and the mixture was heated at $90^{\circ} \mathrm{C}$ for $24 \mathrm{~h}$. At the conclusion of the reaction (TLC monitoring) the DA adduct was oxidized with DDQ (181 $\mathrm{mg}, 0.79 \mathrm{mmol})$ in refluxing toluene $(10 \mathrm{~mL})$ for $36 \mathrm{~h}$. Then, the solvent was removed at reduced pressure and the crude product obtained was purified by flash silica-gel column chromatography with 5\% EtOAc/PE mixture to afford the aromatized product $36(110 \mathrm{mg}, 66 \%)$ as a semi-solid.

\subsection{Physical measurements}

2.2a 1,2-Dimethoxy-4-(octa-1,3,7-trien-2-yl)benzene (11): $\mathrm{R}_{f}=0.74$ (silica gel, petroleum ether) IR (Neat) $v_{\max }: 740,810,1265,1516,1601,2928 \mathrm{~cm}^{-1} ;{ }^{1} \mathrm{H}$ NMR (400 MHz, $\left.\mathrm{CDCl}_{3}\right): \delta 2.15-2.23(\mathrm{~m}, 4 \mathrm{H}), 3.87$ (s, 3H), 3.89 (s, 3H), 4.95-4.99 (m, 2H), 5.05 (d, $J=$ $2.0 \mathrm{~Hz}, 1 \mathrm{H}), 5.14(\mathrm{~d}, J=1.2 \mathrm{~Hz}, 1 \mathrm{H}), 5.67-5.79$ $(\mathrm{m}, 2 \mathrm{H}), 6.30(\mathrm{~d}, J=15.2 \mathrm{~Hz}, 1 \mathrm{H}), 6.83-6.88(\mathrm{~m}, 3 \mathrm{H})$ 
ppm; ${ }^{13} \mathrm{C}$ NMR $\left(100 \mathrm{MHz}, \mathrm{CDCl}_{3}\right): \delta 32.3,33.5,55.9$, 56.0, 110.8, 111.6, 114.2, 114.9, 120.6, 131.9, 133.4, 133.6, 138.2, 147.7, $148.5 \mathrm{ppm}$.

2.2b 1-Choloro-4-(octa-1,3,7-trien-2-yl)benzene (20): $\mathbf{R}_{f}=0.71$ (silica gel, petroleum ether) IR (Neat) $v_{\max }$ : 1011, 1604, 2854, 2925, $3054 \mathrm{~cm}^{-1}$; ${ }^{1} \mathrm{H}$ NMR (400 $\left.\mathrm{MHz}, \mathrm{CDCl}_{3}\right): \delta 2.13-2.31(\mathrm{~m}, 4 \mathrm{H}), 4.95-4.99(\mathrm{~m}, 2 \mathrm{H})$, $5.04(\mathrm{~d}, J=1.2 \mathrm{~Hz}, 1 \mathrm{H}), 5.58-5.62(\mathrm{~m}, 1 \mathrm{H}), 5.76-5.78$ (m, 1H), 6.00-6.03 (m, 2H), 7.22-7.27 (m, 4H) ppm; ${ }^{13} \mathrm{C}$ NMR $\left(100 \mathrm{MHz}, \mathrm{CDCl}_{3}\right): \delta 22.0,23.0,32.4,33.5$, $115.1,115.4,123.5,125.4,126.8,128.4,128.6,129.8$, $131.6,134.0,138.2,139.2,147.1 \mathrm{ppm}$.

2.2c 1-Bromo-4-(octa-1,3,7-trien-2-yl)benzene (21): $R_{f}=0.89$ (silica gel, petroleum ether). IR (Neat) $v_{\max }$ : 741, 835, 1011, 1604, 2854, 2925, $3054 \mathrm{~cm}^{-1},{ }_{1}^{1} \mathrm{H}$ NMR $\left(400 \mathrm{MHz}, \mathrm{CDCl}_{3}\right): \delta 2.15-2.22(\mathrm{~m}, 4 \mathrm{H}), 4.97-5.01$ $(\mathrm{m}, 2 \mathrm{H}), 5.06(\mathrm{~d}, J=2.4 \mathrm{~Hz}, 1 \mathrm{H}), 5.58-5.64(\mathrm{~m}, 1 \mathrm{H})$, 5.66-5.78 (m, 1H), 6.00-6.08 (m, 1H), 6.25-7.36 (m, $1 \mathrm{H}), 7.24\left(\mathrm{dd}, J_{1}=8.4, J_{2}=1.6 \mathrm{~Hz}, 2 \mathrm{H}\right), 7.46(\mathrm{dd}$, $\left.J_{1}=8.8, J_{2}=2.0, \mathrm{~Hz}, 2 \mathrm{H}\right) . \mathrm{ppm} ;{ }^{13} \mathrm{C} \mathrm{NMR}(100 \mathrm{MHz}$, $\left.\mathrm{CDCl}_{3}\right): \delta 22.0,23.0,32.3,33.5115 .1,115.4,121.4$, 123.6, 125.3, 127.2, 128.3, 130.1, 131.3, 131.5, 134.0, 138.1, 139.6, $147.1 \mathrm{ppm}$.

2.2d 1-Iodo-4-(octa-1,3,7-trien-2-yl)benzene (22): $\mathrm{R}_{f}=0.75$ (silica gel, petroleum ether). IR (Neat) $v_{\max }$ : 740, 821, 1265, 1485, 1582, 2925, $3054 \mathrm{~cm}^{-1} ;{ }^{1} \mathrm{H}$ NMR $\left(400 \mathrm{MHz}, \mathrm{CDCl}_{3}\right): \delta 2.16-2.23(\mathrm{~m}, 4 \mathrm{H}), 4.97-5.00$ $(\mathrm{m}, 3 \mathrm{H}), 5.12(\mathrm{~d}, J=0.9 \mathrm{~Hz}, 1 \mathrm{H}), 5.58-5.78(\mathrm{~m}, 1 \mathrm{H})$, $5.79-5.86(\mathrm{~m}, 1 \mathrm{H}), 6.29(\mathrm{~d}, J=15.5 \mathrm{~Hz}, 1 \mathrm{H}), 7.06$ $(\mathrm{d}, J=8.2 \mathrm{~Hz}, 2 \mathrm{H}), 7.67(\mathrm{~d}, J=8 \mathrm{~Hz}, 2 \mathrm{H}) \mathrm{ppm}$; ${ }^{13} \mathrm{C}$ NMR $\left(100 \mathrm{MHz}, \mathrm{CDCl}_{3}\right): \delta 32.4,33.5,78.8,93.0$, $115.1,115.4,130.4,131.5,133.7,134.0,137.2,137.3$, 138.0, 138.2, 140.3, $147.1 \mathrm{ppm}$.

2.2e Ethyl 4-methylene-2-(4-methoxyphenylsulfonamido) deca-5,9-dienoate (23): ${ }^{1} \mathrm{H} \mathrm{NMR}\left(400 \mathrm{MHz}, \mathrm{CDCl}_{3}\right)$ : $\delta 1.18$ (2t, 3H for E\&Z), 2.03-2.06 (m, 2H), 2.12-2.16 $(\mathrm{m}, 2 \mathrm{H}), 2.41(\mathrm{~s}, 3 \mathrm{H}), 2.54-2.59(\mathrm{~m}, 2 \mathrm{H}), 3.91-3.95(\mathrm{~m}$, 2H), 4.02-4.07 (m, 1H), 4.86-5.06 (m, 5H), 5.63-5.68 $(\mathrm{m}, 1 \mathrm{H}), 5.77-5.79(\mathrm{~m}, 1 \mathrm{H}), 5.92-5.93(\mathrm{~m}, 1 \mathrm{H}), 7.26-$ $7.27(\mathrm{~m}, 2 \mathrm{H}), 7.68-7.70(\mathrm{~m}, 2 \mathrm{H}) \mathrm{ppm}$.

2.2f (R)-Tert-butyl-2,2-dimethyl-4-(octa-1,3,7-trien-2yl)oxazolidine-3-carboxylate (24): ${ }^{1} \mathrm{H} \quad \mathrm{NMR} \quad(400$ $\left.\mathrm{MHz}, \mathrm{CDCl}_{3}\right): \delta 1.35(\mathrm{~s}, 9 \mathrm{H}), 1.6-1.8(\mathrm{~m}, 6 \mathrm{H}), 2.1-2.3$ $(\mathrm{m}, 4 \mathrm{H}), 3.75\left(\mathrm{t}, 1 \mathrm{H}, J_{1}=6.4 \mathrm{~Hz}, J_{2}=7.6 \mathrm{~Hz}\right) 4.13$ $\left(\mathrm{t}, 1 \mathrm{H}, J_{1}=8.0 \mathrm{~Hz}, J_{2}=7.2 \mathrm{~Hz}\right) 4.6-4.8(\mathrm{~m}, 1 \mathrm{H})$, $4.9-5.1(\mathrm{~m}, 4 \mathrm{H}), 5.4-5.6(\mathrm{~m}, 1 \mathrm{H}), 5.8-6.2(\mathrm{~m}, 2 \mathrm{H})$ ppm. 2.2g Dimethyl 5-(but-3-enyl)-4'-methoxybiphenyl-3,4dicarboxylate (26): IR (Neat) $v_{\max }: 741,1266,1523$, 1732, $2926 \mathrm{~cm}^{-1} ;{ }_{1}^{1} \mathrm{H}$ NMR (400 MHz, $\left.\mathrm{CDCl}_{3}\right): \delta 2.40$ $\left(\mathrm{dd}, J_{1}=8, J_{2}=1.2, \mathrm{~Hz}, 2 \mathrm{H}\right), 2.75-2.79(\mathrm{~m}, 2 \mathrm{H}) 3.86$ (s, 3H), 3.91 (s, 3H), 3.95 (s, 3H), 4.99-5.01 (m, 2H), 5.80-5.88 (m, 1H), 6.99 (d, $J=4.8, \mathrm{~Hz}, 2 \mathrm{H}), 7.52-7.56$ $(\mathrm{m}, 3 \mathrm{H}), 8.02(\mathrm{~d}, J=1.6 \mathrm{~Hz}, 1 \mathrm{H}) \mathrm{ppm} ;{ }^{13} \mathrm{C}$ NMR $(100$ $\left.\mathrm{MHz}, \mathrm{CDCl}_{3}\right): \delta 35.3,52.5,52.6,55.4,114.4,115.5$. $126.1,128.3,128.7,131.8,133.0,137.5,140.0,141.8$, 159.8, 166.5, $169.8 \mathrm{ppm}$; HRMS(Q-ToF): $\mathrm{m} / \mathrm{z}$ calcd for $\mathrm{C}_{21} \mathrm{H}_{23} \mathrm{O}_{5}[\mathrm{M}+\mathrm{H}]^{+}: 355.1545$ found 355.1537 .

2.2h Dimethyl 3-(but-3-enyl)-5-(4-bromophenyl) cyclohexa-3,5-diene-1,2-dicarboxylate (28): $\quad \mathrm{R}_{f}=0.36$ (silica gel, EtOAc/petroleum ether). IR (Neat) $v_{\max }$ : 740, 1072, 1265, 1605, 1735, $2854 \mathrm{~cm}^{-1} ;{ }^{1} \mathrm{H}$ NMR (400 $\left.\mathrm{MHz}, \mathrm{CDCl}_{3}\right): \delta 2.40\left(\mathrm{dd}, J_{1}=14.8, J_{2}=6.8 \mathrm{~Hz}, 2 \mathrm{H}\right)$, $2.77(\mathrm{t}, J=8.4 \mathrm{~Hz}, 2 \mathrm{H}), 3.91,(\mathrm{~s}, 3 \mathrm{H}), 3.95,(\mathrm{~s}, 3 \mathrm{H})$, 5.02-5.07 (m, 2H), 5.81-5.85 (m, 1H), $7.33(\mathrm{~d}, J=8.4$ $\mathrm{Hz}, 2 \mathrm{H}), 7.58(\mathrm{~d}, J=1.6 \mathrm{~Hz}, 1 \mathrm{H}), 7.79(\mathrm{~d}, J=8.4$ $\mathrm{Hz}, 2 \mathrm{H}), 8.02$ (d, $J=1.2 \mathrm{~Hz}, 1 \mathrm{H}) \mathrm{ppm} ;{ }^{13} \mathrm{C}$ NMR $(100$ $\left.\mathrm{MHz}, \mathrm{CDCl}_{3}\right): \delta 33.1,35.4,52.8,94.3,115.8,126.4$, 128.97, 129.1, 132.1, 134.2, 137.4, 138.3, 139.0, 140.4, $141.2,166.3,169.7 \mathrm{ppm}$.

2.2i Dimethyl 3-(but-3-enyl)-5-(4-iodophenyl) cyclohexa3,5-diene-1,2-dicarboxylate (29): $\mathrm{R}_{f}=0.61$ (silica gel, 5\% EtOAc/petroleum ether). IR (Neat) $v_{\max }: 743$, 1262, 1582, 1738, $2929 \mathrm{~cm}^{-1} ;{ }^{1} \mathrm{H}$ NMR (400 MHz, $\left.\mathrm{CDCl}_{3}\right): \delta 2.40\left(\mathrm{dd}, J_{1}=14.4, J_{2}=6.8 \mathrm{~Hz}, 2 \mathrm{H}\right), 2.77$ (t, $J=8 \mathrm{~Hz}, 2 \mathrm{H}) 3.91$ (s, 3H), 3.95 (s, 3H), 4.99-5.07 (m, 2H), 5.83-5.85 (m, 1H), $7.33(\mathrm{~d}, J=8.4 \mathrm{~Hz}, 2 \mathrm{H})$, $7.58(\mathrm{~d}, J=1.6 \mathrm{~Hz}, 1 \mathrm{H}), 7.79,(\mathrm{~d}, J=8.0 \mathrm{~Hz}, 2 \mathrm{H})$, $8.02(\mathrm{~d}, J=1.2 \mathrm{~Hz}, 1 \mathrm{H}) \mathrm{ppm} ;{ }^{13} \mathrm{C} \mathrm{NMR}(100 \mathrm{MHz}$, $\left.\mathrm{CDCl}_{3}\right): \delta 33.0,35.4,52.8,94.3,115.8,126.4,128.9$, 129.1, 132.1, 134.2, 137.4, 138.3, 139.0, 140.4, 141.7, 169.7 ppm; Anal. Calcd for $\mathrm{C}_{20} \mathrm{H}_{19} \mathrm{IO}_{4}: \mathrm{C}, 53.35 ; \mathrm{H}$, 4.25; found: C, 52.47; H, 3.19.

2.2j Dimethyl 3-(but-3-enyl)-5-ethoxy-2-(4-methoxypheylulfonamido)-3-oxopropyl) phthalate (30): IR (Neat) $v_{\max }: 3055,2985,1737,1266 \mathrm{~cm}^{-1} ;{ }^{1} \mathrm{H}$ NMR $(400 \mathrm{MHz}$, $\left.\mathrm{CDCl}_{3}\right): \delta 1.17(\mathrm{t}, J=7.2 \mathrm{~Hz}, 3 \mathrm{H}), 2.29-2.40(\mathrm{~m}, 5 \mathrm{H})$, 2.58-2.66 (m,1H), $2.99(1 / 2 \mathrm{Abq}, J=13.7,6.7 \mathrm{~Hz}$, $1 \mathrm{H}), 3.10(1 / 2 \mathrm{Abq}, J=13.7,5.5 \mathrm{~Hz}, 1 \mathrm{H}), 3.85-$ $3.93(\mathrm{~m}, 5 \mathrm{H}), 4.11-4.16(\mathrm{~m}, 1 \mathrm{H}), 5.00-5.04(\mathrm{~m}, 2 \mathrm{H})$, $5.20(\mathrm{~d}, J=9 \mathrm{~Hz}, 1 \mathrm{H}), 5.79-5.85(\mathrm{~m}, 1 \mathrm{H}), 7.17-7.23$ $(\mathrm{m}, 3 \mathrm{H}), 7.53-7.59 \mathrm{(m}, 3 \mathrm{H}) \mathrm{ppm} ;{ }^{13} \mathrm{C}$ NMR $(100 \mathrm{MHz}$, $\left.\mathrm{CDCl}_{3}\right): \delta 14.1,21.7,32.8,35.3,39.0,52.7,56.6,62.3$, $115.7,127.2,128.2,128.9,134.1,135.2,136.5,136.5$, 136.7, 137.4, 139.9, 143.9, 166.0, 169.7, 170.7 ppm; 
HRMS(Q-ToF): $m / z$ calcd for $\mathrm{C}_{26} \mathrm{H}_{32} \mathrm{NO}_{8} \mathrm{~S}[\mathrm{M}+\mathrm{H}]^{+}$: 518.1863 found 518.1849 .

$2.2 \mathrm{k}$ (4R)-Tert-butyl-4-(4-(but-3-enyl)-9,10-dioxo-1,4, 4a,9,9a,10-hexahydroanthracen-2-yl)-2,2-Dimethyloxazolidine-3-carboxylate (31): ${ }^{1} \mathrm{H}$ NMR (400 MHz, $\left.\mathrm{CDCl}_{3}\right): \delta 1.28(\mathrm{~s}, 9 \mathrm{H}), 1.4-1.6(\mathrm{~m}, 6 \mathrm{H}), 1.6-2.2$ (m, 9H), 3.7-3.9 (m, 1H), 4.1-4.2 (m, 1H), 4.35-4.45 $(\mathrm{m}, 1 \mathrm{H}), 4.9-5.1(\mathrm{~m}, 2 \mathrm{H}), 5.76-5.80(\mathrm{~m}, 2 \mathrm{H}), 7.7-7.9$ (m, 2H), 8.1-8.3 (m, 2H) ppm.

2.21 Dimethyl 4'-cholorobiphenyl-3, 4-dicarboxylate (37): $\mathrm{R}_{f}=0.85$ (silica gel, 5\% EtOAc/petroleum ether). IR (Neat) $v_{\max }: 740,1266,1497,1731,2954 \mathrm{~cm}^{-1}$; ${ }^{1} \mathrm{H}$ NMR (400 MHz, $\left.\mathrm{CDCl}_{3}\right): \delta 3.93(\mathrm{~s}, 3 \mathrm{H}), 3.94(\mathrm{~s}$, $3 \mathrm{H}), 7.44\left(\mathrm{dd}, J_{1}=6.8, J_{2}=2.0 \mathrm{~Hz}, 3 \mathrm{H}\right), 7.54$ dd, $\left.J_{1}=6.8, J_{2}=2.0 \mathrm{~Hz}, 2 \mathrm{H}\right), 7.70\left(\mathrm{dd}, J_{1}=8\right.$, $\left.J_{2}=2.0 \mathrm{~Hz}, 1 \mathrm{H}\right), 7.85\left(\mathrm{dd}, J_{1}=8.0, J_{2}=1.6 \mathrm{~Hz}, 1 \mathrm{H}\right)$ ppm; ${ }^{13} \mathrm{C} \mathrm{NMR}\left(100 \mathrm{MHz}, \mathrm{CDCl}_{3}\right): \delta 52.8,52.9,127.3$, $127.4,128.6,129.4,130.3,133.3,134.9,137.5,143.2$, 167.6, $168.3 \mathrm{ppm}$.

$2.2 \mathrm{~m}$ Dimethyl 4'-bromobiphenyl-3,4-dicarboxylate (38): $\quad \mathbf{R}_{f}=0.38$ (silica gel, EtOAc/petroleum ether). IR (Neat) $v_{\max }: 743,819,1265,1734,2852 \mathrm{~cm}^{-1} ;{ }^{1} \mathrm{H}$ NMR (400 MHz, $\left.\mathrm{CDCl}_{3}\right): \delta 3.92(\mathrm{~s}, 3 \mathrm{H}), 3.94(\mathrm{~s}, 3 \mathrm{H})$, $7.45(\mathrm{~d}, J=2.4 \mathrm{~Hz}, 2 \mathrm{H}), 7.60(\mathrm{~d}, J=1.6 \mathrm{~Hz}, 2 \mathrm{H})$, $7.70\left(\mathrm{dd}, J_{1}=8, J_{2}=1.8 \mathrm{~Hz}, 1 \mathrm{H}\right), 7.85\left(\mathrm{dd}, J_{1}=9.2\right.$, $\left.J_{2}=2 \mathrm{~Hz}, 2 \mathrm{H}\right) \mathrm{ppm} ;{ }^{13} \mathrm{C} \mathrm{NMR}\left(100 \mathrm{MHz}, \mathrm{CDCl}_{3}\right)$ : $\delta 52.8,52.9,123.0,127.3,128.9,129.2,129.9,130.4$, 132.3, 133.3, 138.0, 143.2, 167.6, 168.2 ppm; HRMS (Q-ToF): $\mathrm{m} /$ z calcd for $\mathrm{C}_{16} \mathrm{H}_{13} \mathrm{BrO}_{4}[\mathrm{M}+\mathrm{H}]^{+}: 349.0078$ found 349.0075 .

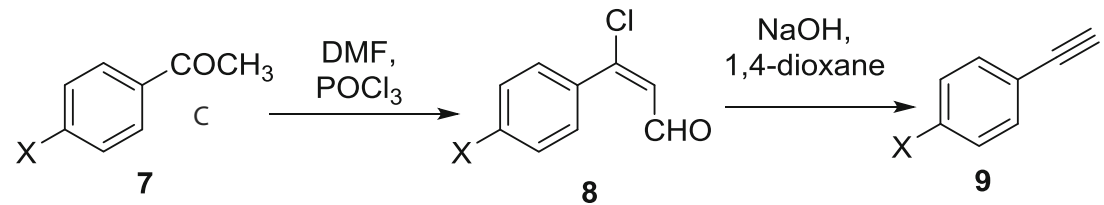

Scheme 1. Preparation of various phenyl acetylene derivatives.<smiles>C#Cc1ccc(OC)c(OC)c1</smiles>

Scheme 2. Preparation and DA reaction of homoallyl 1, 3-butadiene derivatives.

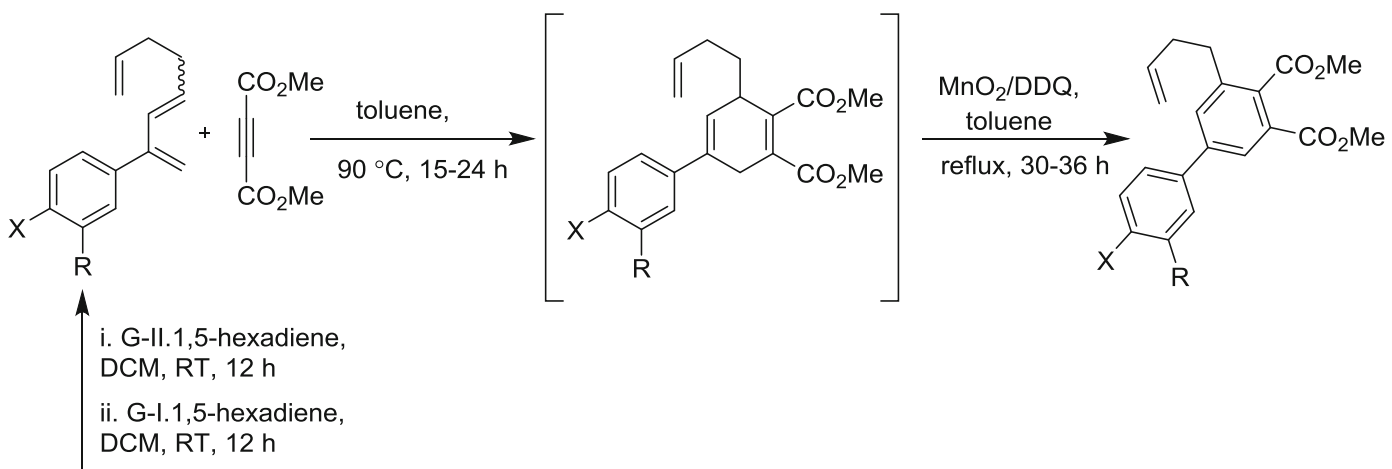

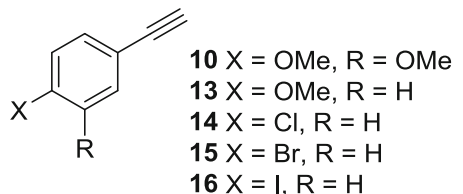

$16 X=I, R=H$

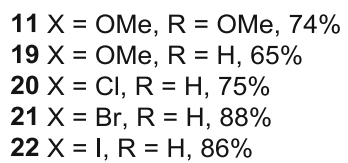

$25 X=O M e, R=O M e, 75 \%$

$26 X=O M e, R=H, 66 \%$

$27 X=\mathrm{Cl}, \mathrm{R}=\mathrm{H}, 60 \%$

$28 X=B r, R=H, 72 \%$

$29 X=I, R=H, 82 \%$

Scheme 3. General strategy for the synthesis of homoallyl 1, 3-butadiene derivatives and their DA reaction. 


\section{Results and Discussion}

Recently, Kotha and Seema reported a preliminary communication involving the application of cross-enyne metathesis (CEM) and the DA reaction as key steps for assembling the biaryl derivatives. ${ }^{9}$ Herein, we report the full details about the preparation of biaryls and two amino acid derivatives as additional examples. ${ }^{10}$

Table 1. Preparation of homoallyl 1,3-dienes and aromatized/DA products.

1<smiles>C#Cc1ccc(OC)c(OC)c1</smiles>

10

2<smiles>C#Cc1ccc(OC)cc1</smiles>

13

3<smiles>C#Cc1ccc(Cl)cc1</smiles>

14<smiles>C#Cc1ccc(Br)cc1</smiles>

15<smiles>C#Cc1ccc(I)cc1</smiles>

16<smiles>C#CCC(N[Na])C(=O)OCC</smiles>

17

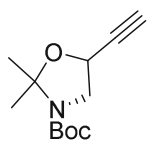

18
Product

Product<smiles>C=CCC/C=C\C(=C)c1ccc(OC)c(OC)c1</smiles>

$11(74 \%)$<smiles>C=CCC/C=C\C(=C)c1ccc(OC)cc1</smiles>

$19(65 \%)$<smiles>C=C(/C=C\CC/C=C\C)c1ccc(Cl)cc1</smiles>

$$
20(75 \%)
$$

$\mathrm{Br}$

$$
21(88 \%)
$$<smiles>C=C(/C=C\CCC)c1ccc(I)cc1</smiles>

$22(86 \%)$<smiles>C=CC(=CC(=O)OCC)CC(N)C(=O)OCC</smiles>

$$
23(63 \%)
$$<smiles>C=C(/C=C\CCC)C1CCC(C)(C)O1</smiles>

$24(81 \%)$<smiles>C=CCCc1cc(-c2ccc(OC)c(OC)c2)cc(C(C)=O)c1C(C)=O</smiles>

$25(75 \%)$<smiles>C=CCCc1cc(-c2ccc(OC)cc2)cc(C(C)=O)c1C(C)=O</smiles>

$26(66 \%)$<smiles>CC=CCc1cc(-c2ccc(Cl)cc2)cc(C(C)=O)c1C(C)=O</smiles>

$27(60 \%)$<smiles>CC(=O)c1cc(C(C)=O)c(-c2cccc(Br)c2)cc1CC/C=C\c1ccc(Br)cc1</smiles><smiles>C=CCCc1cc(-c2ccc(I)cc2)cc(C(C)=O)c1C(C)=O</smiles>

$29(82 \%)$<smiles>C=CCCc1cc(CC(N[13N])OCC)cc(C(C)=O)c1C(C)=O</smiles>

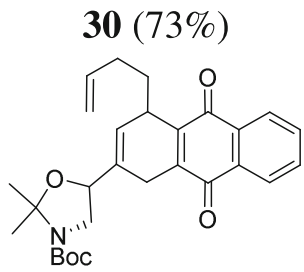

31 (52\%) 
Toward the synthesis of biaryl derivatives, commercially available acetophenones were chosen as starting materials. These acetophenones 7 were converted to aryl acetylenes by the Vilsmeier formylation followed by the base treatment (scheme 1). ${ }^{11}$

Initially, CEM was performed starting with 3,4dimethoxyphenyl acetylene and 4-methoxyphenyl acetylene (10 and 13) using 1,5-hexadiene as a crosscoupling partner in the presence of the Grubbs Second generation (G-II) catalyst under toluene reflux (scheme 2). Under these conditions cross-enyne product $\mathbf{1 1}$ has been generated and later it was converted to the corresponding 3,4-dimethoxy biaryl system 12 by intramolecular metathesis and aromatization sequence.

When the same reaction was carried out in toluene at $90^{\circ} \mathrm{C}$, an inseparable mixture of cis-trans isomers of $\mathbf{1 1}$ was formed in $74 \%$ yield. ${ }^{12}$ Similarly, halogen containing aryl acetylenes 14-16 were subjected to CEM with 1,5-hexadiene using the Grubbs first generation catalyst (G-I) in DCM at RT under nitrogen (scheme 3). Again, inseparable mixture of cis-trans isomers of diene was obtained in good yield. Since the dienes generated are converted to corresponding aromatic products by the DA reaction and aromatization sequence; the geometry of the double bond in the diene is of no consequence.

When these dienes were subjected to the DA reaction with dienophile such as dimethyl acetylenedicarboxylate (DMAD) $)^{13}$ in toluene at $90^{\circ} \mathrm{C}$ for $24 \mathrm{~h}$, the formation of the cycloadduct was observed (TLC monitoring). The DA adduct formed was directly subjected to aromatization sequence with $\mathrm{MnO}_{2}$ in 1,4-dioxane, and also using DDQ in toluene. It was observed that the reaction using DDQ in refluxing toluene gave better yield of the aromatized products (table 1). To expand the scope of the CEM methodology, we have prepared acetylene derivatives such as $\mathbf{1 7}$ and $\mathbf{1 8}$ containing $\alpha$-amino acid moiety. The preparation of $\mathbf{1 7 ^ { 1 4 }}$ and $\mathbf{1 8},{ }^{15}$ started with readily available starting materials such as glycine and serine by using the known methodology. The acetylenes building blocks $\mathbf{1 7}$ and $\mathbf{1 8}$ were subjected to CEM with 1,5-hexadiene in the presence of G-I. The resulting dienes $\mathbf{2 3}$ and $\mathbf{2 4}$ were subjected to the DA reactions with DMAD and napthoquinone respectively. We observed that napthoquinone DA adduct was not converted to the corresponding aromatized product even after prolonged heating in toluene at $100^{\circ} \mathrm{C}$.

In the second strategy, the compound $\mathbf{1 3}$ was subjected to cross-metathesis in the presence of ethylene ( $1 \mathrm{~atm}$ pressure) by using the Grubbs second generation catalyst in toluene at RT. However, it was found that at $90^{\circ} \mathrm{C}$, the required building block was obtained in an acceptable yield. ${ }^{16}$ Halogenated phenylacetylene derivatives 14-16 delivered the required dienes in the presence of G-II in DCM at RT for $24 \mathrm{~h}$ (scheme 4). The disappearance of acetylene portion and the appearance of new peaks at $\delta 5.12-5.29 \mathrm{ppm}$ and at $\delta$ $6.66 \mathrm{ppm}$ in ${ }^{1} \mathrm{H}$ NMR spectroscopy indicate the formation of diene. These experiments indicated that toluene is a better solvent for cross-enyne metathesis under ethylene atmosphere as well as 1,5-hexadiene conditions with substrates such as 4-methoxy and 3,4dimethoxy phenylacetylenes. DCM seems to be a better solvent for halogenated phenylacetylene substrates for cross-metathesis under ethylene or 1,5-hexadiene conditions. The dienes obtained during cross-metathesis sequence were subjected to the DA reaction with the dienophile such as DMAD under toluene reflux conditions, to deliver the corresponding DA adducts. Since we observed that these dienes are prone to polymerize under toluene reflux conditions, the DA reaction was performed in toluene at $90^{\circ} \mathrm{C}$ for $14-24 \mathrm{~h}$ that gave better yield of the DA adducts (TLC monitoring). The
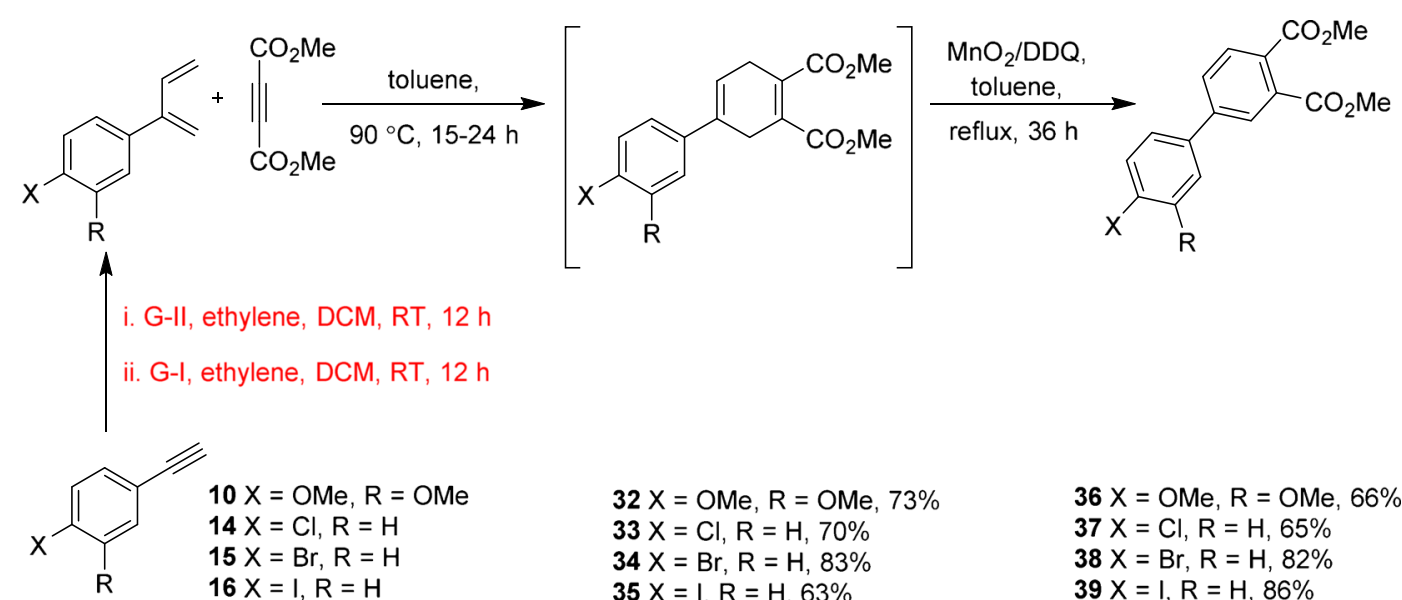

$$
\begin{aligned}
& 32 X=O M e, R=O M e, 73 \% \\
& 33 X=C l, R=H, 70 \% \\
& 34 X=B r, R=H, 83 \% \\
& 35 X=I, R=H, 63 \%
\end{aligned}
$$

$$
\begin{aligned}
& 36 X=O M e, R=O M e, 66 \% \\
& 37 X=C l, R=H, 65 \% \\
& 38 X=B r, R=H, 82 \% \\
& 39 X=I, R=H, 86 \%
\end{aligned}
$$

Scheme 4. General strategy for the synthesis of 1, 3-butadiene derivatives and their DA reaction. 
Table 2. List of compounds prepared by CEM, DA reaction and aromatization sequence.

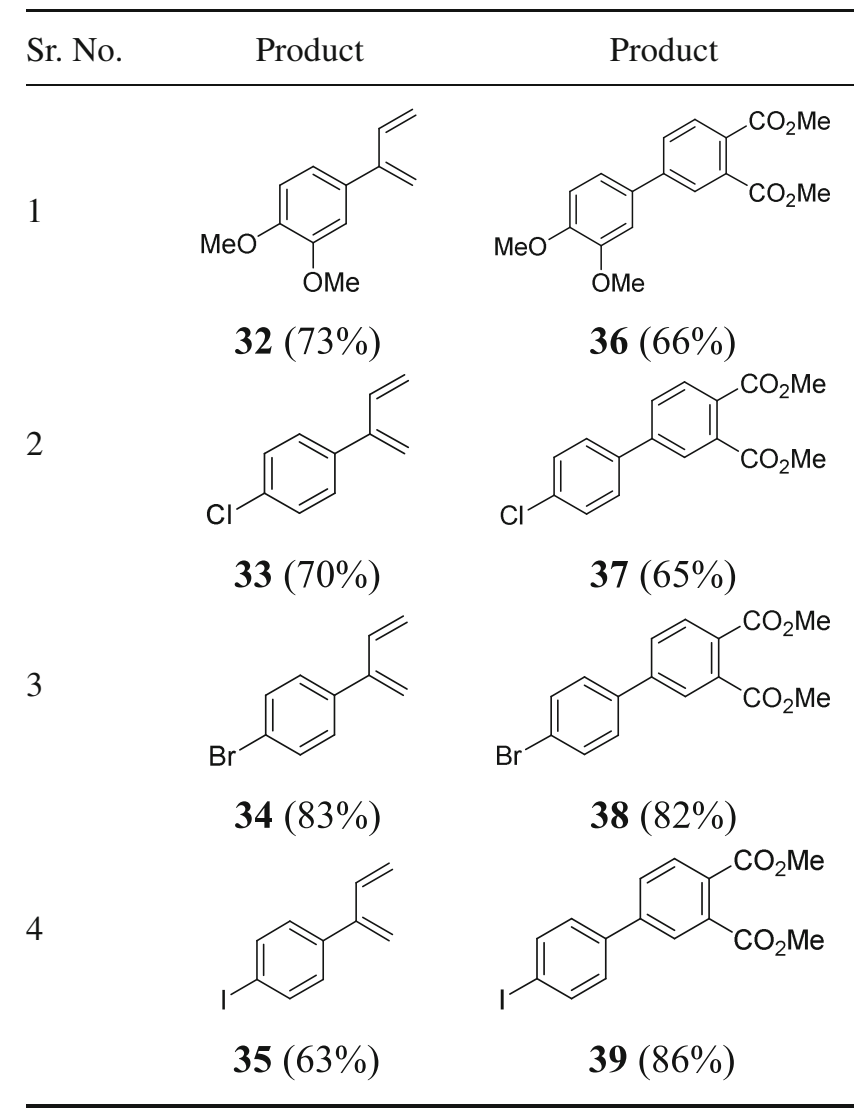

DA products were further subjected to aromatization sequence using DDQ under refluxing toluene to deliver the aromatized products (table 2).

\section{Conclusions}

We have demonstrated that cross-enyne metathesis of acetylene derivatives in the presence of 1,5-hexadiene or ethylene followed by a Diels-Alder reaction and aromatization sequence furnished polycyclics. The generality of this methodology has been established with a variety of acetylene derivatives. Moreover, this methodology offers several diversity points. The first option is to choose a variety of acetylene derivatives. The second option involves the selection of various partners during olefin cross-metathesis and the third option is to use dienophiles during the DA reaction. The olefin present at the end of the metathesis sequence offers an additional handle for further synthetic manipulation.

\section{Supplementary Information}

${ }^{1} \mathrm{H}$ and ${ }^{13} \mathrm{C}$ NMR (Figures $\mathrm{S} 1$ to $\mathrm{S} 10$ ) are given in Supplementary Information available free of charge in www.ias.ac.in/chemsci.

\section{Acknowledgements}

We would like to acknowledge the DST for the financial support. We also thank, SAIF-Mumbai for recording the spectral data. S.V. thanks IIT-Bombay and UGCNew Delhi for the award of a research fellowship. We thank Dr. D. Deodhar for his help in preparing the manuscript. S. K. thanks DST for the award of J C Bose Fellowship.

\section{References}

$\dagger$ Major portion of the work was taken from Seema V, Ph.D. thesis, IIT-Bombay 2011

1. (a) Bringmann G, Hinrichs J, Henschel P, Kraus J and Peters E M 2002 Eur. J. Org. Chem. 1096; (b) Baudoin O 2005 Eur. J. Org. Chem. 4223; (c) Mark S and Butler M S 2004 J. Nat. Prod. 672141

2. (a) McGlacken G P and Bateman L M 2009 Chem. Soc. Rev. 38 2447; (b) Waldmann H, He Y P, Tan H, Arveab L and Arndt H D 2008 Chem. Commun. 5562

3. Donato L, Mourot A, Davenport C M, Herbivo C, Warther D, Lonard J, Bolze F, Nicoud J F, Kramer R H, Goeldner M and Specht A 2012 Angew. Chem. Int. Ed. 511840

4. Hassan J, Sevignon M, Gozzi C, Schulz E and Lemaire M 2002 Chem. Rev. 1021359

5. (a) Cepanec I 2004 In Synthesis of Biaryls (New York: Elsevier); (b) Bates R 2012 In Organic Synthesis using Transition Metals (UK: John Wiley)

6. King O, Okukado N and Negishi E J 1977 J. Chem. Soc. Chem. Commun. 683

7. Van de Kuil L A, Grove D M, Gossage R A, Zwikker J W, Jenneskens L W, Drenth, W and Koten G V 1997 Organometallics 164985

8. (a) Shahzad S A, Vivant C and Wirth $\mathrm{T} 2010$ Org. Lett. 12 1364; (b) Esmaeili A A, Moradi A and Mohammadi H K 2010 Tetrahedron. 66 3575; (c) Ashburn B O and Carter R G 2008 Org. Biomol. Chem. 6 255; (d) Ashburn B O and Carter R G 2006 Angew. Chem. Int. Ed. 45 6737; (e) Becht J M and Drian C L 2008 Org. Lett. 10 3161; (f) Pearson A J and Bum Kim J B 2003 Tetrahedron Lett. 44 8525

9. Kotha S and Seema V 2011 Synlett 2329

10. (a) Kotha S, Misra S and Srinivas V 2012 Eur. J. Org. Chem. 4052; (b) Kotha S, Goyal D, Thota N and Srinivas V 2012 Eur. J. Org. Chem. 1843; (c) Kotha S and Lahiri K 2007 Synlett 2767; (d) Kotha S, Meshram M and Tiwari A 2009 Chem. Soc. Rev. 38 2065; (e) Kotha S, Mandal K, Banerjee S and Mobin S M 2007 Eur. J. Org. Chem. 1244

11. Kotha S, Tafesh A, Davenport K and Ortiz P 2001 Indian J. Chem. Sect. B 401166

12. Banerjee S 2007 Synthetic approaches to novel polycyclics via the Diels-Alder reaction (PhD Thesis: IIT Bombay, India) 
13. (a) Kotha S and Khedkar P 2008 Synthesis 2925; (b) Jason A, Smulik S T and Diver S 2000 Org. Lett. 2 2271

14. O'Donnell M J and Plott R A 1982 J. Org. Chem. 47 2663
15. Sreenivasachary N 2000 Design of new building for the synthesis of unusual $\alpha$-amino acid derivatives $(\mathrm{PhD}$ Thesis: IIT Bombay, India)

16. Kotha S and Khedkar P 2009 J. Org. Chem. 74 5667 